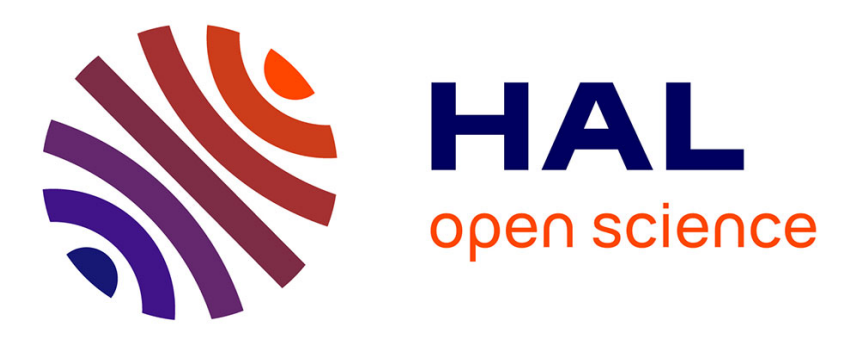

\title{
A fast analytical method to compute the radial flux density distribution in the airgap of a superconducting inductor
}

Gaël Malé, Smail Mezani, Thierry Lubin, Jean Lévêque

\section{- To cite this version:}

Gaël Malé, Smail Mezani, Thierry Lubin, Jean Lévêque. A fast analytical method to compute the radial flux density distribution in the airgap of a superconducting inductor. IEEE Transactions on Applied Superconductivity, 2010, 10.1109/TASC.2010.2096172 . hal-00537666

\section{HAL Id: hal-00537666 https://hal.science/hal-00537666}

Submitted on 19 Nov 2010

HAL is a multi-disciplinary open access archive for the deposit and dissemination of scientific research documents, whether they are published or not. The documents may come from teaching and research institutions in France or abroad, or from public or private research centers.
L'archive ouverte pluridisciplinaire HAL, est destinée au dépôt et à la diffusion de documents scientifiques de niveau recherche, publiés ou non, émanant des établissements d'enseignement et de recherche français ou étrangers, des laboratoires publics ou privés. 


\title{
A fast analytical method to compute the radial flux density distribution in the airgap of a superconducting inductor
}

\author{
Gaël Malé, Student Member, IEEE, Smail Mezani, Thierry Lubin and Jean Lévèque
}

\begin{abstract}
An original topology of superconducting rotating machine propose to use the diamagnetism of high temperature superconducting (HTS) bulk. Here, YBCO bulks allow to shield the magnetic field created by two solenoids supplied by dc currents in contra-directions. The obtained airgap field is multipolar as in conventional ac machines. A quick analytical method is developed here to compute the magnetic field distribution. It is based on the determination of the modulating function associated with the radial flux density distribution resulting from the introduction of the superconducting bulks. A first step allows the calculation of the modulating function by a 2D analytical method (using the resolution of Laplace's and Poisson's equations by the separation of variables technique). The later is used in a second step together with the field produced by the two solenoids to calculate the radial flux density distribution in the airgap of the considered inductor. Comparisons to experiments and to $3 \mathrm{D} F E$ results show the validity of the proposed approach with the benefit of low computation time.
\end{abstract}

Index Terms-Analytical solution, superconducting rotating machines, superconducting materials, magnetic shield.

\section{INTRODUCTION}

$S^{\mathrm{t}}$ UPERCONDUCTING MATERIALS are used in different electrical devices like motors and generators which represent an important part of the superconducting applications [1] such as marine propulsion, offshore wind generators. Moreover, the shielding properties of these materials gave rise to a variety of electrical machine topologies.

Actually in some types of electrical machines (claw pole, Lyndell machines...) ferromagnetic materials are used to modulate and concentrate the magnetic field to obtain a multipolar wave in the air-gap from a constant excitation. In superconducting (SC) applications, ferromagnetic materials are heavily saturated since they are subjected to high magnetic fields. Hence, they can not be used for modulation purposes.

Therefore the SC shields can be used as flux barriers in variable reluctance motors [2] to increase the saliency ratio or to allow flux concentration. From this last idea, an original topology of inductor, Fig.1, has been proposed [3] and a SC synchronous motor has been designed, built and successfully tested [4].

Several methods like Monte Carlo methods [5,6] or 3D finite elements can be used to compute the magnetic field distribution in this 3D structure but this leads to a very large computation time.

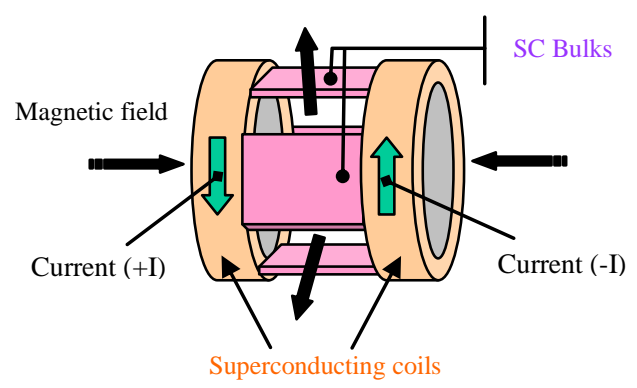

Fig. 1. Schematic of the flux concentration SC inductor.

Another approach presented in this paper is based on the determination of the modulating function associated with the radial flux density distribution resulting from the introduction of the SC bulks. Comparisons to experiments and to 3D FE results show the validity of the proposed approach with the benefit of huge savings in computation time.

\section{PROBLEM DESCRIPTION AND ASSUMPTIONS}

Figure 1 shows the studied SC inductor. It uses SC bulks, which present the specificity to have a diamagnetic behavior under zero-field cooling, to shield the magnetic field created by two solenoids supplied by dc currents in contra-directions. The obtained airgap field is multipolar as in conventional ac machines. This flux density is minimal above a SC bulk and maximal above the bulks interspaces.

In the present work, a $2 \mathrm{D}$ domain corresponding to the median cross section of the $\mathrm{SC}$ inductor (taken at $\mathrm{z}=0$ ) is considered. The aim being the determination of the radial flux density distribution in the air gap. The following assumptions are made:

- HTS bulks are modeled with radial sides.

- Perfect diamagnetic behavior of the HTS bulks under zero-field cooling.

The last assumption is equivalent as to impose a Dirichlet condition on the surfaces of the superconducting bulks for the magnetic vector potential

$$
A=0
$$

This is justified by the fact that superconductors in normal operation perfectly shield the magnetic field. However, it is obvious that the study of the real behavior of a superconductor in term of current density distribution under applied external magnetic field is of great importance. Indeed, this allows the determination of the needed geometrical parameters of the SC bulk to "do the job" i.e. to shield the external field. 
Now, this paper considers that the SC bulk is effectively behaving like a screen against the external magnetic field.

In the following, a method for computing the radial flux density created by the inductor of Fig. 1 is presented.

\section{PRINCIPLE OF THE MethoD}

At a given axial position in the air above the inductor, the radial flux density distribution at a radial distance $r$ produced by the two solenoids can be written as:

$$
B_{r}(r, \theta)=B_{r 0}(r) \cdot \Lambda_{r}(r, \theta)
$$

where $\theta$ is the angular position, $B_{r 0}$ is the radial flux density created by the solenoids without the SC bulks and $\Lambda_{r}$ is the modulating function.

The model of the SC inductor is split into two steps. Firstly, $B_{r 0}$ is computed analytically using the Biot-Savart law [7]. Secondly, the modulating function which only depends on the geometry and on the material properties of the system is written as follows:

$$
\Lambda_{r}(r, \theta)=\Lambda_{r 0}(r)+\sum_{k=1,2,3 \ldots} \Lambda_{r k}(r) \cos (k Q \theta)
$$

where $\Lambda_{r k}$ are the Fourier coefficients for the modulating function and $Q$ is the number of superconducting bulks.

From (2) and (3):

$$
B_{r}(r, \theta)=B_{r 0}(r) \Lambda_{r 0}(r)+\sum_{k=1,2,3 \ldots} B_{r 0}(r) \Lambda_{r k}(r) \cos (k Q \theta)
$$

From (4), one can see that the radial flux density exhibits a dc component which doesn't create any "emf". The number of pole-pairs of the device is $p=Q$ since the highest space harmonic is obtained for $k=1$. $B_{r 0}$ being known, we have to determine the modulating function $\Lambda_{r}$ at a given axial position to get the radial flux density distribution using (4).

\section{A. Determination of the modulating function}

To calculate $\Lambda_{r}$, let us consider a $2 \mathrm{D}$ domain corresponding to the median cross section of Fig.1 (taken at $z=0$ ).

Firstly, the SC bulks are removed. At a radius $R_{l}$, Fig. 2a, we impose a tangential magnetic field of the form

$$
h_{s}\left(R_{1}, \theta\right)=H_{0} \cos (p \theta)
$$

$H_{0}$ can take any value and the magnetic field distribution is easily calculated by an analytical approach. From this computation, the obtained radial flux density at a radius $R_{e}$ is called $B_{r a}\left(R_{e}, \theta\right)$.

In a second step, the SC bulks are introduced, Fig. $2 b$. The same condition on $R_{I}$ as in (5) is used. The radial flux density resulting from the introduction of the SC bulks (details in the next sub-section) is called $B_{r b}\left(R_{e}, \theta\right)$

Finally, the modulating function is simply obtained by

$$
\Lambda_{r}\left(R_{e}, \theta\right)=\frac{B_{r b}\left(R_{e}, \theta\right)}{B_{r a}\left(R_{e}, \theta\right)}
$$

\section{B. Analytical formulation to determine $B_{r b}$}

The studied geometry is show in Fig.3. A magnetic vector

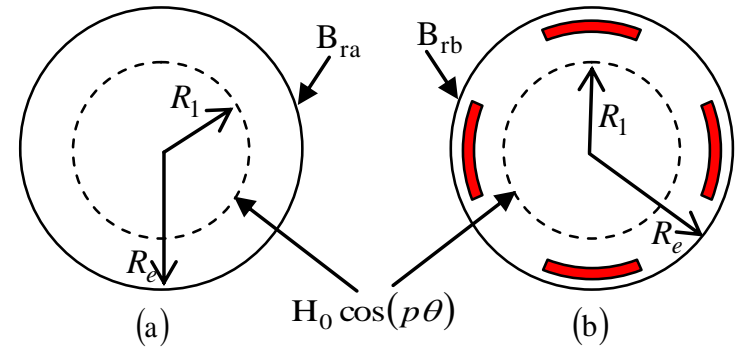

Fig. 2. Studied domains for the determination of the modulating function (a): without SC bulks and (b): including SC bulks

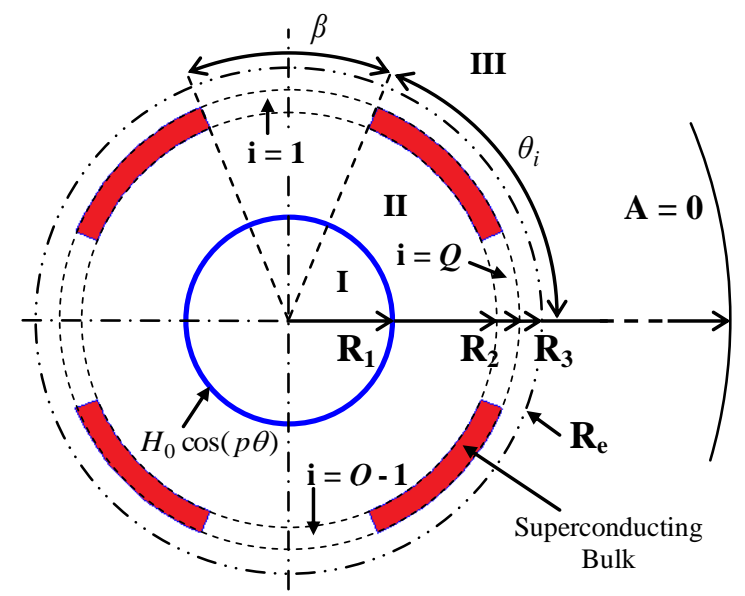

Fig. 3. Studied geometry to determine the modulating function $(Q=4)$

potential formulation has been chosen in 2D polar coordinates to describe the problem. According to the adopted assumptions, the magnetic vector potential has only one component along the $z$-direction and only depends on the $r$ and $\theta$ coordinates.

Laplace's equation is solved in the holes (inter bulk space) and in the different air sub-domains. The separation of variables technique is employed to get the solution.

1) General Solution in the $i$-th hole sub-domain (regions $i=1$ to $i=Q$ )

In the $i$-th hole sub-domain, the Laplace equation in polar coordinates is written as

$$
\frac{\partial^{2} A_{i}}{\partial r^{2}}+\frac{1}{r} \frac{\partial A_{i}}{\partial r}+\frac{1}{r^{2}} \frac{\partial^{2} A_{i}}{\partial \theta^{2}}=0 \text { for }\left\{\begin{array}{l}
R_{2} \leq r \leq R_{3} \\
\theta_{i} \leq \theta \leq \theta_{i}+\beta
\end{array}\right.
$$

Considering a perfect diamagnetic behavior for the HTS bulks, the normal component of the magnetic field at the sides of the HTS bulk is null. The boundary conditions for the $i$-th hole domain are

$$
\left.A_{i}\right|_{\theta=\theta_{i}}=0 \text { and }\left.A_{i}\right|_{\theta=\theta_{i}+\beta}=0
$$

The continuity of the tangential component of the magnetic field between the $i$-th hole and its neighboring air sub-domains (regions II and III) leads to

$$
\left.\frac{\partial A_{i}}{\partial r}\right|_{r=R_{1}}=\left.\frac{\partial A_{I}}{\partial r}\right|_{r=R_{1}} \text { and }\left.\frac{\partial A_{i}}{\partial r}\right|_{r=R_{2}}=\left.\frac{\partial A_{I I}}{\partial r}\right|_{r=R_{2}}
$$

The general solution of (7) is found by the method of the separation of variables [8] (described by a Sturm-Liouville problem). Using (8), it is written as 


$$
A_{i}(r, \theta)=\sum_{n=1}^{\infty}\left(C_{n}^{i} \cdot r^{-\frac{n \pi}{\beta}}+D_{n}^{i} \cdot r^{\frac{n \pi}{\beta}}\right) \cdot \sin \left(\frac{n \pi}{\beta}\left(\theta-\theta_{i}\right)\right)
$$

where $C_{n}^{i}$ and $D_{n}^{i}$ are constants to be determined using the interface conditions (9).

\section{2) General solution in sub-domain I}

The problem to solve is

$$
\frac{\partial^{2} A_{I}}{\partial r^{2}}+\frac{1}{r} \frac{\partial A_{I}}{\partial r}+\frac{1}{r^{2}} \frac{\partial^{2} A_{I}}{\partial \theta^{2}}=0 \text { for }\left\{\begin{array}{l}
0 \leq r \leq R_{1} \\
0 \leq \theta \leq 2 \pi
\end{array}\right.
$$

The boundary condition at $r=R_{1}$ is

$$
A_{I}\left(R_{1}, \theta\right)=A_{I I}\left(R_{1}, \theta\right)
$$

The general solution of (11) is well known [8] (periodic Sturm-Liouville problem in a disc). Taking into account the boundary condition (12) and the fact that the magnetic vector potential must be finite at $r=0$, the general solution of (11) is

$$
A_{I}(r, \theta)=A_{0}^{I}+\sum_{h=1}^{\infty}\left(\frac{r}{R_{1}}\right)^{h}\left(A_{h}^{I} \cdot \cos (h \theta)+C_{h}^{I} \cdot \sin (h \theta)\right)
$$

where $h$ is a positive integer. The coefficients $A_{0}^{I}, A_{h}^{I}$ and $C_{h}^{I}$ are determined using a Fourier series expansion of $A_{I I}\left(R_{1}, \theta\right)$ over the interval $[0,2 \pi]$

3) General solution in sub-domain II and III

In the sub-domains II and III, the problem to solve is

$$
\frac{\partial^{2} A_{X}}{\partial r^{2}}+\frac{1}{r} \frac{\partial A_{X}}{\partial r}+\frac{1}{r^{2}} \frac{\partial^{2} A_{X}}{\partial \theta^{2}}=0 \text { for }\left\{\begin{array}{l}
R_{i_{X}} \leq r \leq R_{e_{X}} \\
0 \leq \theta \leq 2 \pi
\end{array}\right.
$$

where $X$ corresponds to sub-domain (II or III) and $R_{i_{X}}$ and $R_{e_{X}}$ are respectively the inner and the outer radii of each subdomain.

In sub-domain II, the boundary condition at $r=R_{1}$ is

$$
\left.\frac{\partial A_{I}}{\partial r}\right|_{r=R_{1}}-\left.\frac{\partial A_{I I}}{\partial r}\right|_{r=R_{1}}=\mu_{0} \cdot h_{s}
$$

The continuity of the magnetic vector potential at the interface between the holes and sub-domain II ( $\left.r=R_{2}\right)$ leads to

$$
A_{\text {II }}\left(R_{2}, \theta\right)=F(\theta)
$$

And considering that the magnetic vector potential is equal to zero elsewhere (diamagnetic proprieties of the superconducting bulks), one can write

$$
F(\theta)= \begin{cases}A_{i}\left(R_{2}, \theta\right) & \forall \theta \in\left[\theta_{\mathrm{i}}, \theta_{i}+\beta\right] \\ 0 & \text { elsewhere }\end{cases}
$$

where $A_{i}(r, \theta)$ is the magnetic vector potential in the $i$-th hole given by (10).

In the sub-domain III, the boundary condition at $r=R_{4}$ is

$$
A_{\text {III }}\left(R_{4}, \theta\right)=0
$$

The radius $R_{4}$ is chosen far enough to not affect the results.

The same method as the one presented for sub-domain II is used to determine the boundary condition at $r=R_{3}$.
Introducing the function $G(\theta)$, the boundary condition can be written as

$$
A_{\text {III }}\left(R_{3}, \theta\right)=G(\theta)
$$

with

$$
G(\theta)= \begin{cases}A_{i}\left(R_{3}, \theta\right) & \forall \theta \in\left[\theta_{\mathrm{i}}, \theta_{i}+\beta\right] \\ 0 & \text { elsewhere }\end{cases}
$$

where $A_{i}(r, \theta)$ is the magnetic vector potential in the $i$-th hole given by (10).

Finally, the general solution of (14) is well known [8] (periodic Sturm-Liouville problem in an annulus). By taking into account the boundary conditions (15), (16) in II and (18), (19) in III the general solution of the magnetic vector potential in the air-gaps can be written as

$$
\begin{aligned}
& A_{X}(r, \theta)=A_{0}^{X}+B_{0}^{X} \cdot \ln (r) \\
& +\sum_{k=1}^{\infty}\left(A_{k}^{X} \cdot r^{-k}+B_{k}^{X} \cdot r^{k}\right) \cdot \cos (k \theta) \\
& +\sum_{k=1}^{\infty}\left(C_{k}^{X} \cdot r^{-k}+D_{k}^{X} \cdot r^{k}\right) \cdot \sin (k \theta)
\end{aligned}
$$

where $k$ is a positive integer. The coefficients $A_{0}^{X}, B_{0}^{X}$, $A_{k}^{X}, B_{k}^{X}, C_{k}^{X}$ and $D_{k}^{X}$ are determined using Fourier series expansions of the boundary conditions over the interval $[0$, $2 \pi]$.

The radial flux density at $r=R_{e}$ is

$$
B_{r b}(r, \theta)=\left.\frac{1}{r} \frac{\partial A_{I I I}}{\partial \theta}\right|_{r=R_{e}}
$$

\section{SimUlation StUdies}

An inductor (IND1) is considered with characteristics given in Table I. This inductor is a part of a synchronous machine which has been constructed and successfully tested in our laboratory [4]. This inductor was designed to create a 4 polepairs airgap flux density so it consists of 4 YBCO bulks, each of them being made from assembling 2 rectangular SC tapes.

\section{A. Modulating function}

Figure 4 shows the obtained modulating function and its harmonic spectrum at $r=124 \mathrm{~mm}$. As expected from (2), the presence of the SC bulks results in a number of harmonics, the largest of which is the dc component followed by the $4^{\text {th }}$ harmonic.

TABLE I

PARAMETERS OF SC INDUCTOR (IND1)

\begin{tabular}{|l|l|}
\hline Solenoid SC material (wires) & $\mathrm{NbTi}$ \\
\hline Number of solenoids & 2 \\
\hline Inner radius of solenoid, $\mathrm{mm}$ & 75 \\
\hline Outer radius of solenoid, $\mathrm{mm}$ & 105 \\
\hline Axial length of solenoid, mm & 50 \\
\hline Axial distance between solenoids, mm & 50 \\
\hline Bulk SC material & YBCO \\
\hline Number of bulks $(Q)$ & 4 \\
\hline Inner radius of bulk, mm & 100 \\
\hline Bulk thickness, mm & 5 \\
\hline Bulk axial length, mm & 50 \\
\hline Bulk angular opening, deg & 45 \\
\hline
\end{tabular}



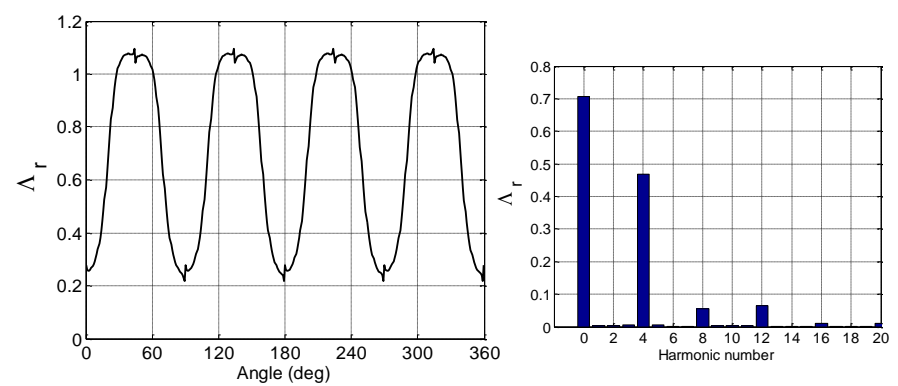

Fig. 4. Modulating function and its harmonic spectrum $(r=124 \mathrm{~mm})$

Notice that in conventional smooth airgap ac machines, the inductor field (created by windings or permanent magnets) is multipolar with a nil mean value (no dc component).

\section{B. Flux density waveforms}

The variation of the radial flux density at $r=124 \mathrm{~mm}$ is shown in Fig. 5 together with its harmonic spectrum.

The NbTi solenoids have been supplied by direct current of about $15 \mathrm{~A}$ which leads to a current density $J=70 \mathrm{~A} / \mathrm{mm}^{2}$. The radial flux density created by the solenoids when the SC bulks are removed is about $B_{r 0}=0.38 T$. The resulting radial flux density $B_{r}(r, \theta)$ is calculated using (2). Clearly, the presence of the SC screens results in a number of space harmonics, the largest of which corresponds to 4 pole-pairs. Hence, the number of pole-pairs of the armature windings must be equal to 4 to create the highest possible torque.

To validate the proposed method, 3D FE analyses have been carried out using commercial software (COMSOL MULTIPHYSICS $^{\circledR}$ ). To obtain acceptable results, we used a mesh of about 250000 tetrahedral elements with a vector potential formulation.

A good agreement is noticed between the FE results and those issued from the proposed method, Fig.6a. The computation time is about 3 hours with the 3D FE analysis whereas it takes only few seconds with the proposed approach. It is clear that in the first design stage of the inductor where multiple computations are needed, the proposed model can be advantageously used.

The study of the axial dependence of the radial flux density assumes that the modulating function doesn't depend on the axial coordinate $z$. Hence, the same modulating function of Fig.4 has been used for the computations. The radial flux density is computed using (3) with the difference that $B_{r 0}$ computed with the Biot-Savart law depends now on $z$. Again, the agreement is good between the different methods in use, Fig. 6 b.
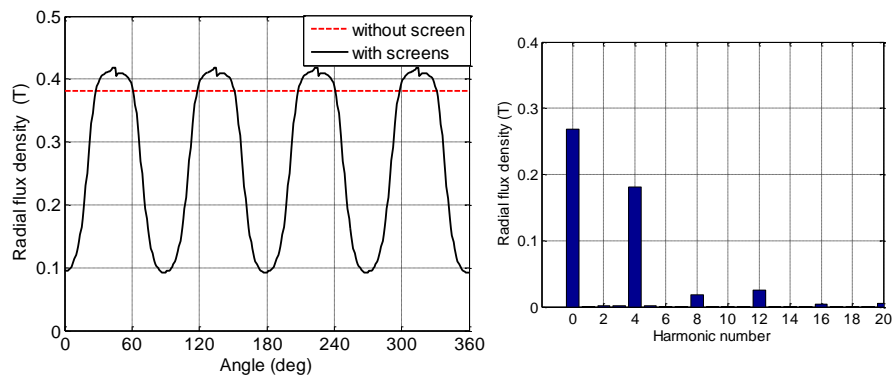

Fig. 5. Radial flux density waveform and its harmonic spectrum $(r=124 \mathrm{~mm}$, $\left.J=70 \mathrm{~A} / \mathrm{mm}^{2}\right)$

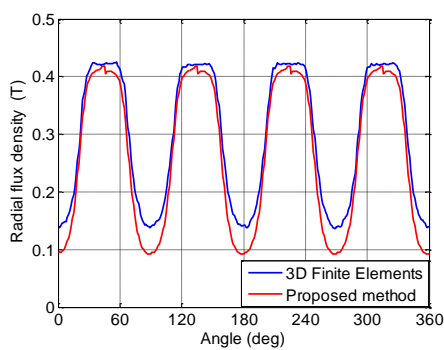

(a)

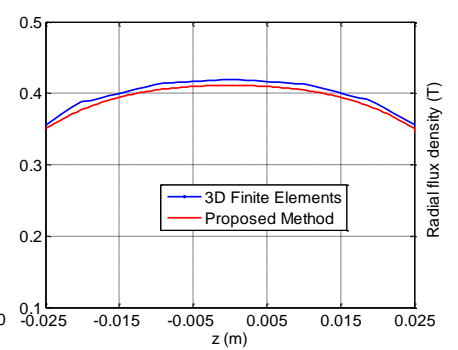

(b)
Fig. 6. Comparison of radial flux densities ( $\left.r=124 \mathrm{~mm}, J=70 \mathrm{~A} / \mathrm{mm}^{2}\right)$

(a) along $\theta$-direction, (b) axial dependence taken between the bulks interspaces

\section{COMPARISONS TO EXPERIMENTS}

To corroborate the simulation studies, the obtained results have been compared to some experimental data.

Another inductor has been built in our laboratory [3]. Its characteristics are given in Table II. The flux density versus the angular position has been measured with ten Hall probes numbered from S1 to S10, Fig.7.

Figure 8 presents a comparison between the measured flux densities and the calculated ones. It can be seen that the results issued from our method are in good accordance with the measurements and with the 3D FE results as well.

\section{CONCLUSION}

A simple and quick method to compute the radial flux density created by an original topology of a superconducting inductor has been presented. Comparisons to 3D finite element analyses and to experimental results show the validity of the proposed approach with the benefit of huge savings in computation time.

TABLE II

PARAMETERS OF SC INDUCTOR (IND2)

\begin{tabular}{|l|l|}
\hline Solenoid SC material (wires) & $\mathrm{NbTi}$ \\
\hline Number of solenoids & 2 \\
\hline Inner radius of solenoid, $\mathrm{mm}$ & 20 \\
\hline Outer radius of solenoid, $\mathrm{mm}$ & 32 \\
\hline Axial length of solenoid, mm & 45 \\
\hline Axial distance between solenoids, $\mathrm{mm}$ & 30 \\
\hline Bulk SC material & YBCO \\
\hline Number of bulks $(Q)$ & 4 \\
\hline Inner radius of bulk, mm & 23 \\
\hline Bulk thickness, $\mathrm{mm}$ & 5 \\
\hline Bulk axial length, $\mathrm{mm}$ & 25 \\
\hline Bulk angular opening, deg & 57 \\
\hline
\end{tabular}

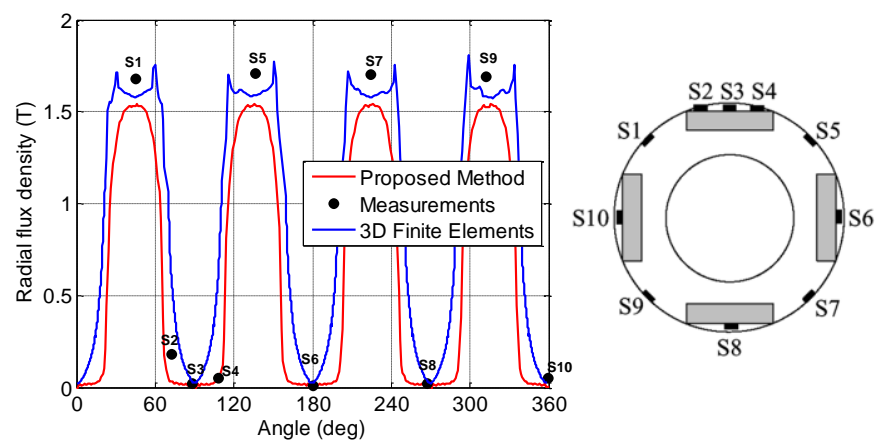

Fig. 7. Comparison of calculated and measured flux densities $(r=32 \mathrm{~mm}$, $I=260 A$ ) 


\section{REFERENCES}

[1] R. Schiferl, A. Flory, W. C. Livoti and S. D. Umans, "High-Temperature Superconducting Synchronous Motors: Economic Issues for Industrial Applications," IEEE Trans. Appl. Superconduct., vol. 44, No 5, pp. 1376-1384, Sept.-oct. 2008.

[2] L. K. Kovalev, and all, "Hysteresis and reluctance electric machines with bulk HTS elements. Recent results and future development," Supercond. Sci. Technol., vol. 13, pp. 498-502, 2000.

[3] P. Masson, J. Lévêque, D. Netter, A. Rezzoug, "Experimental study of a new kind of superconducting inductor," IEEE Trans. on App. Supercon., vol. 13(2), pp. 2239-2242, 2003.

[4] E. Ailam, D. Netter, J. Lévêque, B. Douine, A. Rzzoug, P. Masson, "Design and testing of a superconducting motor," IEEE Trans. on App. Supercon., vol. 17(1), pp. 27-33, 2007.
[5] S. Mandayam, L. Udpa, S. S. Udpa and W. Lord, "Monte Carlo methods for modelling magnetostatic NDE phenomena: A feasibility study," IEEE Trans. Mag., vol. 32(3), pp. 1425-1428, 1996.

[6] P. Masson, J. Lévêque, D. Netter, A. Rezzoug, "Magnetic field concentration: Comparison between several shapes of superconducting shields," IEEE Trans. on App. Supercon., vol. 11(1), pp. 2248-2251, 2001.

[7] A. Rezzoug, J. P. Caron, F. M. Sargos, "Analytical calculations of flux induction and forces of thick coils with finite length," IEEE Trans. Magn., vol. 28(5), pp. 2250-2252, 1992.

[8] S. J. Farlow, Partial Differential Equations for Scientists and Engineers, New York: Dover, 1993. 